\title{
Relationship of the fabella with the origins of the plantaris and gastrocnemius lateral head muscles in late-term fetuses: a histological study
}

\author{
Zhe-Wu Jin ${ }^{1}$, Ji Hyun Kim ${ }^{2}$, Daisuke Suzuki ${ }^{3}$, Namiko Sugai $^{4}$, Gen Murakami ${ }^{5}$, Hiroshi Abe ${ }^{6}$, \\ José Francisco Rodríguez-Vázquez ${ }^{7}$ \\ ${ }^{1}$ Department of Anatomy, Wuxi School of Medicine, Jiangnan University, Wuxi, Jiangsu, China, ${ }^{2}$ Department of Anatomy, Jeonbuk National University \\ Medical School, Jeonju, Korea, ${ }^{3}$ Divison of Common Curriculum, Hokkaido Chitose College of Rehabilitation, Chitose, ${ }^{4}$ Divison of Rehabilitation, \\ Hitsujigaoka Hospital of Orthopedics, Sapporo, ${ }^{5}$ Division of Internal Medicine, Cupid Clinic, Iwamizawa, Hokkaido, ${ }^{6}$ Emeritus Professor of Akita \\ University School of Medicine, Akita, Japan, ${ }^{7}$ Department of Anatomy and Embryology, School of Medicine, Complutense University, Madrid, Spain
}

\begin{abstract}
Previous studies of midterm fetuses indicated that a cartilaginous fabella appeared to be embedded in the plantaris $(\mathrm{PL})$, and was fused with the gastrocnemius lateral head (GL). We re-examined the topographical anatomy of the fabella or its analogue (a tight fibrous mass) originating in the GL and/or PL by evaluating histological sections of the unilateral knees of 15 late-term fetuses. Regardless of whether the cartilaginous fabella was present (6 fetuses) or absent ( 9 fetuses), the origins of the PL and GL muscles each had three parts. In each fetus, the fabella or its analogue was embedded in a thick common tendinous origin of the GL and PL. PL1 (whose origin is similar to that of the adult PL) originated from the femoral condyle immediately above the common tendon; PL2 originated from the posteromedial aspect of the fabella or its analogue; and PL3 originated from the inferior aspect of the fabella or its analogue. The muscle fibers of PL1, PL2, and PL3 joined to provide a thick plantaris. GL1 (which is adjacent to PL2) originated from the common tendon in the superior side of the fabella or its analogue and GL2 originated from the inferior side of the fabella or its analogue. GL1 and GL2 joined to provide a thick bundle, whereas GL3 (located far below the fabella or its analogue) originated from the posterior surface aponeurosis. Therefore, drastic reconstruction at these muscle origins was necessary during development. Due to the strong mechanical stress from the GL and the space-occupying effect of the muscle, we hypothesize that PL2 and PL3 are degraded or absorbed into the GL1 and GL2 during the postnatal period, so that the remaining PL1 was likely the remaining PL in adults.
\end{abstract}

Key words: Plantaris muscle, Gastrocnemius muscle, Fabella, Knee, Human fetus

Received December 31, 2020; Revised February 10, 2021; Accepted March 12, 2021

Corresponding author:

Ji Hyun Kim (1D

Department of Anatomy, Jeonbuk National University Medical School, Jeonju 54907, Korea

E-mail:407kk@hanmail.net

\section{Introduction}

Previous examination of the histological sections of midterm fetuses with or without fabellas showed that the fetal plantaris (PL) muscle was as large as the gastrocnemius lateral head (GL) and that the fabella or its analogue (a tight fibrous mass) was embedded in the origin of the PL rather than the GL [1]. The position of this analogue is identical to that of the cartilaginous fabella, but they differ in immuno- 
reactivity. These findings, and consideration of adult morphology, indicated the fetal fabella moved from the origin of the PL to that of the GL. Therefore, the morphology of the GL and PL are different in fetuses and adults, especially at their origins. Other studies also reported differences in fetal and adult muscle morphology in the forearm [2], elbow [3], and posterior thigh [4]. However, our previous hypothesis proposed a drastic reconstruction of muscle origins from the lateral condyle of the femur.

Consequently, we examined late-term fetuses in the present study to determine whether the fabella is present at the origin of the PL or GL. We hypothesized that the morphology of the fabella and related muscles of late-term fetuses would be transient, and intermediate between the morphology of midterm fetuses and adults.

\section{Materials and Methods}

The study was performed in accordance with the provisions of the 1995 Declaration of Helsinki, as revised in 2013. Sagittal and longitudinal histological sections of the knees of 15 late-term human fetuses (gestational age [GA]: 28-37 weeks; crown-rump length [CRL]: 228-310 mm) were prepared. These fetuses, which were in a collection at the Department of Anatomy, Akita University (Japan) were donated by their families from 1975 to 1985 and preserved in 10\% w/w neutral formalin solution for more than 30 years. Information was available on the date of donation and GA, but not on family name, name of the obstetrician or hospital, and the reason for abortion. The use of these samples for research was approved by the Akita University Ethics Committee (no. 1378).

After separating each unilateral knee from the body, the specimens were incubated at room temperature in PlankRychlo solution $\left(\mathrm{AlCl}_{2} / 6 \cdot \mathrm{H}_{2} \mathrm{O}, 7.0 \% \mathrm{w} / \mathrm{v} ; \mathrm{HCl}, 3.6 \% \mathrm{w} / \mathrm{v}\right.$; $\mathrm{HCOOH}, 4.6 \% \mathrm{w} / \mathrm{v}$ ) for 1 to 2 weeks. The specimens were then embedded in paraffin using routine procedures, and semi-serial sagittal sections (0.1-0.2 mm intervals) were prepared, depending on the size of the specimen. All tissue sections were stained with hematoxylin and eosin (H\&E). The posterolateral muscles at the knee, including those in lateterm specimens, were identified as described previously [5].

\section{Results}

\section{Multiple muscle bundles at the origins}

Six of the 15 analyzed knees contained cartilaginous fabellas (Figs. 1,2) and the other 9 contained tight fibrous masses instead (Figs. 3-5). In both groups, the fabella or its analogue was embedded in a short but thick tendinous band originating from the femoral lateral condyle on the superomedial side of the popliteus tendon insertion (Figs. 1A, B, $2 \mathrm{~A}, 3 \mathrm{~A}, \mathrm{~B}, 4 \mathrm{~A})$. Thus, the tendinous band was similar to the most proximal part of the adult GL. However, the origins of the GL and PL were each consistently composed of 3 muscle bundles (GL1, GL2, and GL3; PL1, PL2, and PL3; Table 1), regardless of the presence of a cartilaginous fabella. The upper origin of the PL (PL1) was muscular rather than tendinous, and was similar to the PL origin in adults. The tendinous band is the common tendon because parts of the GL and PL originated from it. The distance from the insertion of the common tendon to the insertion of the popliteus tendon at the lateral condyle varied among specimens, from less than $1 \mathrm{~mm}$ (Fig. 1A, B) to more than $5 \mathrm{~mm}$ (Fig. 4A). However, there is some uncertainty in these measurements because the two insertions were not contained in any single sagittal section. The three GL bundles and the 3 PL bundles joined to provide an independent thick muscle bundle, whereas a single specimen with a fabella analogue apparently had complete fusion between the GL and PL at the knee joint level, a structure we consider to be an anomaly (Fig. 5). There was striation of muscle fibers at each of the 6 origins, although our demonstration of this was limited (Fig. 2I).

The origin of the PL was consistently composed of the upper (PL1), middle (PL2), and lower (PL3) bundles. PL1, which is independent from the common tendon and from PL2 and PL3, originated from the femoral condyle immediately above the common tendon (Figs. 1D, E, 2D, 3B, C, 4A). Analysis of the origins of the 6 bundles of the GL and PL indicated that only one (PL1) had a "muscular" origin, in which muscle fibers appeared to originate directly from the femoral condyle. PL2 originated from the posteromedial aspect of the common tendon more than $2 \mathrm{~mm}$ below PL1 (Figs. 1G, 2G, 3E, $4 \mathrm{D}, \mathrm{E}$ ), whereas PL3 originated from the inferior end (Figs. $1 \mathrm{~F}, 2 \mathrm{~F}, 3 \mathrm{D}, 4 \mathrm{E})$. Thus, PL2 and PL3 appeared to originate from the cartilaginous fabella when it was present. Striated muscle fibers were not directly attached to the cartilage, but intermingled with the fibrous capsule. The muscle fibers of PL1, PL2, and PL3 joined to provide a thick muscle belly of 

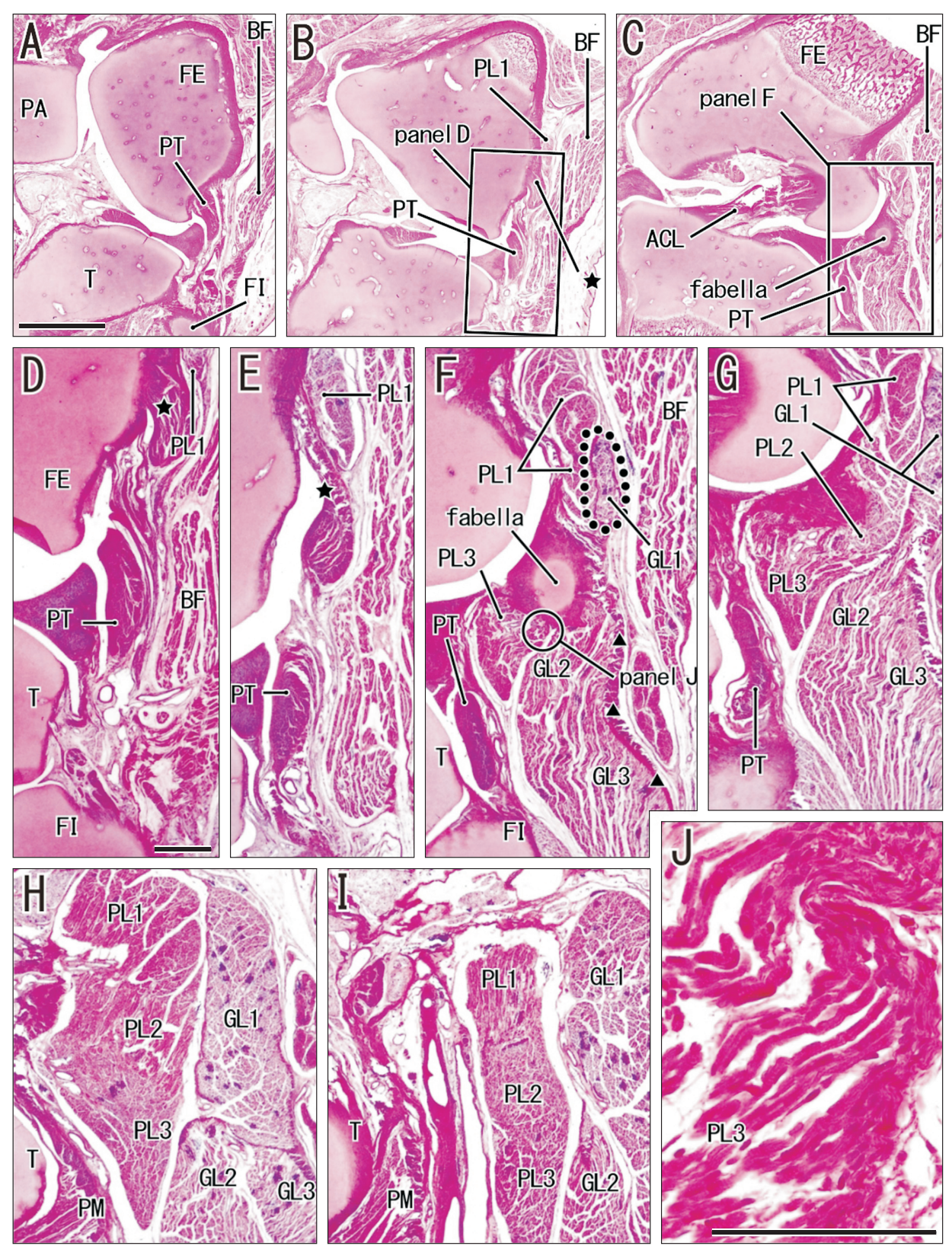

Fig. 1. Origins of the gastrocnemius lateral head (GL) and plantaris (PL) in a fetus (260 mm crown-rump length [CRL]) with a fabella. (A-C) show the topographical anatomy of bony elements and muscle attachments in the lateral half of the knee joint; (A) shows the most lateral site and (C) the most medial site. (A) shows the insertion of the PT to the femur (FE). (B) shows a common tendinous origin (star) of the GL and PL. (C) shows the fabella and anterior cruciate ligament. (D-I) show multiple muscle bundles at the origins of the GL and PL; (D) shows the most lateral site and (I) the most medial site. (D) corresponds to the square in (B), and (F) corresponds to the square in (C). (D, E) show the upper bundle of the PL (PL1) is adjacent to the posterior aspect of the common tendinous origin (stars). (F, G) show the fabella is surrounded by the PL origin, which has three muscle masses (PL1, PL2, PL3). (F) shows the upper muscle bundle of the GL (GL1, dotted oval) originates from the common tendon, and is adjacent to the inferior aspect of the PL1. (G) shows that after the GL1 combined with another muscle bundle originating from the fabella (GL2), the lateral head was separated from the PL, the lower most muscle bundle (GL3) originates from a surface aponeurosis (triangles in F) that extended from the GL2. (J) (a magnified view of the circle in F) shows the PL3 from the fabella. (A-C) are at low magnification (H\&E, scale bar in A: $5 \mathrm{~mm}),(\mathrm{D}-\mathrm{I})$ are at intermediate magnification $(\mathrm{H} \& \mathrm{E}$, scale bar in $\mathrm{D}: 1 \mathrm{~mm})$, and $(\mathrm{J})$ is at high magnification (H\&E, scale bar: $0.1 \mathrm{~mm}$ ). See also Table 1. PA, patella; BF, biceps femoris; FI, fibula; T, tibia; ACL, anterior cruciate ligament; PM, popliteus muscle; PT, popliteus tendon. 

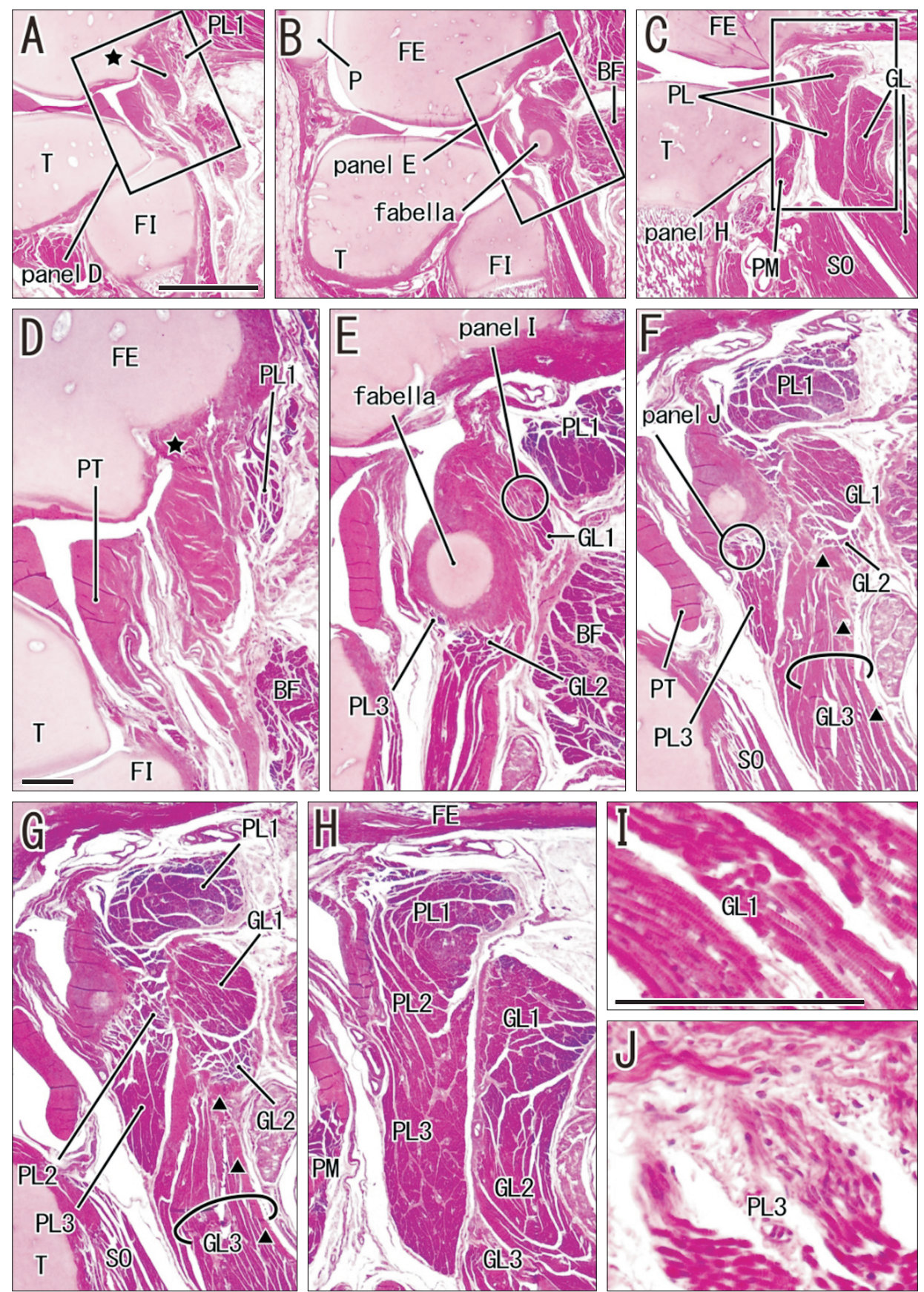

Fig. 2. Origins of the gastrocnemius lateral head (GL) and plantaris (PL) in a fetus (328 mm crown-rump length [CRL]) with a fabella. (A-C) display the topographical anatomy of bony elements and muscle attachments in the lateral half of the knee joint; (A) shows the most lateral site and (C) the most medial site. (A) shows a common tendinous origin (star) of the GL and (B) shows the fabella. (D-H) show multiple muscle bundles at origins of these two muscles. (D, E, H) are magnified views of the squares in $(A-C)$, respectively. (D) shows the most lateral site and $(\mathrm{H})$ the most medial site. (D) shows that the PL1 attaches to the posterior aspect of the common tendinous origin (star). (E, F) show the origin of the GL1, which is distant from the PL1 and originates from the common tendon near fabella, but the GL3 originates from a surface aponeurosis (triangles). (I, J) show muscle fibers of the GL1 and PL3, and are higher magnifications of the circles in (E, F), respectively. (A-C) are at low magnification (H\&E, scale bar in A: $5 \mathrm{~mm}),(\mathrm{D}-\mathrm{H})$ are at intermediate magnification (H\&E, scale bar in D: $1 \mathrm{~mm}$ ), and (I, J) is at high magnification (H\&E, scale bar: $0.1 \mathrm{~mm}$ ). See also Table 1. BF, biceps femoris; FI, fibula; T, tibia; FE, femur; PM, popliteus muscle; PT, popliteus tendon; SO, soleus. 

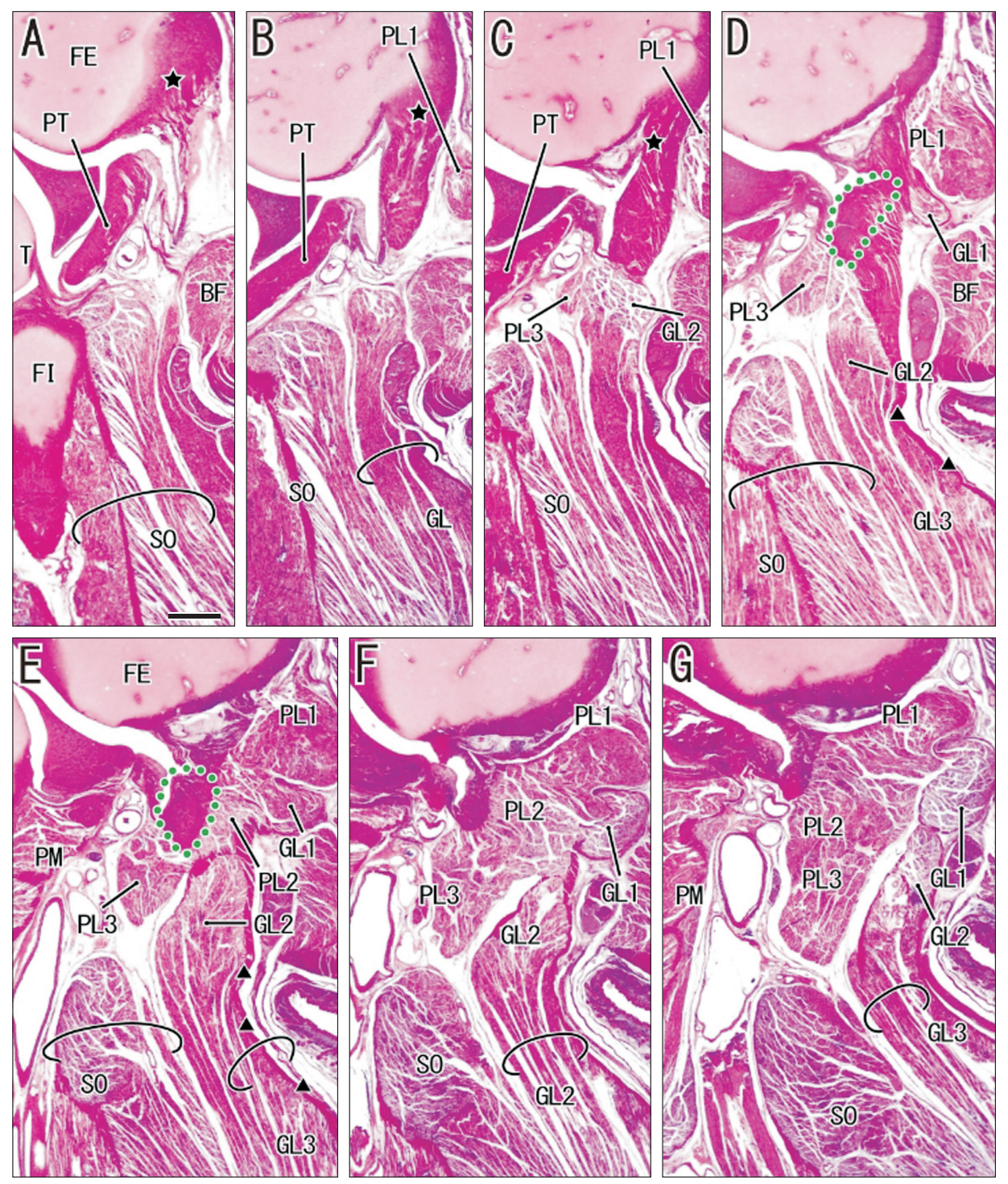

Fig. 3. Origins of the gastrocnemius lateral head (GL) and plantaris (PL) in a fetus ( $250 \mathrm{~mm}$ crown-rump length [CRL]) without a fabella. Panels show multiple muscle bundles at the origins of the GL and PL; (A) shows the most lateral site and (G) the most medial site. (D, E) show a fabella-like tight fibrous mass (green-dotted oval) instead of a fabella at the inferomedial end of a common tendinous origin (star) and (AC) show this fibrous mass is surrounded by four muscle bundles (GL1, GL2, PL2, PL3). (E) shows a posterior surface aponeurosis (triangles) issuing from the lower muscle fibers of the lateral head (GL3). (G) shows the GL1 is separated from the PL1 and PL3 in the far medial side of the popliteus tendon, facing the joint cavity (PT). All panels are at the same magnification (H\&E, scale bar in A: $1 \mathrm{~mm}$ ). See also Table 1. BF, biceps femoris; FI, fibula; FE, femur; PM, popliteus muscle; PT, popliteus tendon; SO, soleus.

the PL, whose thickness was almost the same as that of the GL or the popliteus muscle belly (Figs. 1F, 2H).

Except for a single specimen, the GL was composed of upper (GL1), middle (GL2), and lower (GL3) bundles. GL1 was a large and independent bundle that originated from the common tendon, immediately on the upper side of the fabella or its analogue (Figs. 1F, 2E, 3E, 4B). This bundle extended inferomedially below PL1 to join GL2, and was usually separated from PL1 (Figs. 2E, 3D, 4B) but rarely adjacent to it (Fig.
1F). GL2 originated from the inferior aspect of the fabella or common tendon (Figs. 1F, 2F, 3C, D, 4C), indicating it was located in front of and adjacent to PL2 and PL3. A thick muscle bundle after the joining of GL1 and GL2 (1-2 mm below the fabella or its analogue) had a posterior surface aponeurosis from which GL3 originated (Figs. 1F, 2F, 3F, 4D, E). Thus, GL3 was distant from the common tendon or fabella, GL1 was adjacent to the anteromedial aspect of PL2, and GL2 was inferoposterior to PL3. GL1, GL2, and/or PL1 pushed the 

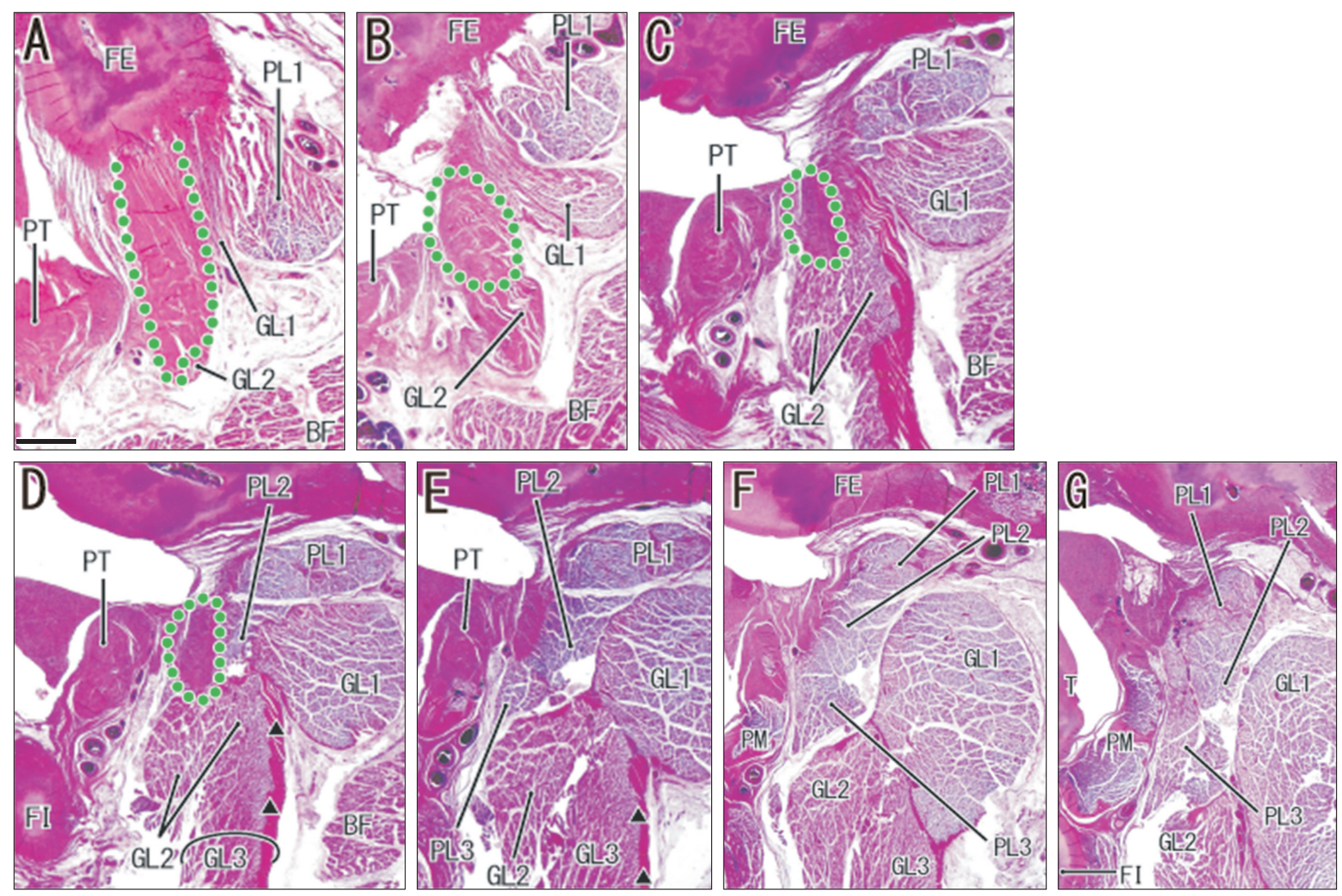

Fig. 4. Origins of the gastrocnemius lateral head (GL) and plantaris (PL) in a fetus (282 mm crown-rump length [CRL]) without a fabella. Panels show multiple muscle bundles at origins of the GL and PL; (A) shows the most lateral site and (G) the most medial site. (A) shows that the common tendinous origin of the GL and PL (dotted green line) is continuous to a fabella-like connective tissue mass (dotted green line in B, C) and these structures are surrounded by multiple muscle bundles (PL1, PL2, PL3, GL1, GL2). (E, F) show another origin of the PL3 appears in the medial side of the fabella-like mass. (F, G) shows the GL1 and GL2 fused to provide a thick muscle in this medial section. (D, E) show the GL3 originates from the surface aponeurosis (triangles). All panels are at the same magnification (H\&E, scale bar in A: $1 \mathrm{~mm}$ ). See also Table 1 . BF, biceps femoris; FI, fibula; FE, femur; PM, popliteus muscle; PT, popliteus tendon.

biceps femoris posteriorly, so it was near the insertion at the knee (Figs. 2E, 3D, 4B, C). Table 1 summarizes our observations of these multiple muscle origins. Notably, the morphology of the gastrocnemius medial head in each midterm fetus was similar to that in adults.

\section{Different muscle origins in specimens with and without fabellas}

PL1, PL2, and PL3 joined to provide the thick muscle belly of the PL, whereas GL1 and GL2 joined to provide the muscle belly of the GL. Lateral sections that contained the popliteus tendon facing the joint cavity indicated that PL3 originated from the inferior aspect of the fabella (Figs. 1F, 2F). Examination of fetuses lacking fabellas indicated that PL3 appeared at the far medial side of the popliteus tendon. Likewise, lateral sections that contained the popliteus tendon indicated that
GL1 was separate from PL2 in originating from the fabella (Figs. 1G, 2G). In the absence of a fabella, medial sections containing the popliteus muscle belly showed that the GL and PL were separate (Figs. 3G, 4F). Therefore, the separation between the GL and PL was more medial in specimens without a fabella. We also observed a well-developed medial head of the gastrocnemius in medial sections (not shown).

\section{An exceptional specimen with fusion between the GL and $P L$}

The common tendon was absent in one fetus $(274 \mathrm{~mm}$ CRL; Fig. 5). Rather, PL1, PL2, and PL3 had a belt-like arrangement on the immediate posterior side of the popliteus tendon (Fig. 5E, F). The GL1 attached to the posterior aspect of the thick muscle bundle at the PL origins, and the GL3 originated from an aponeurosis that extended inferiorly 

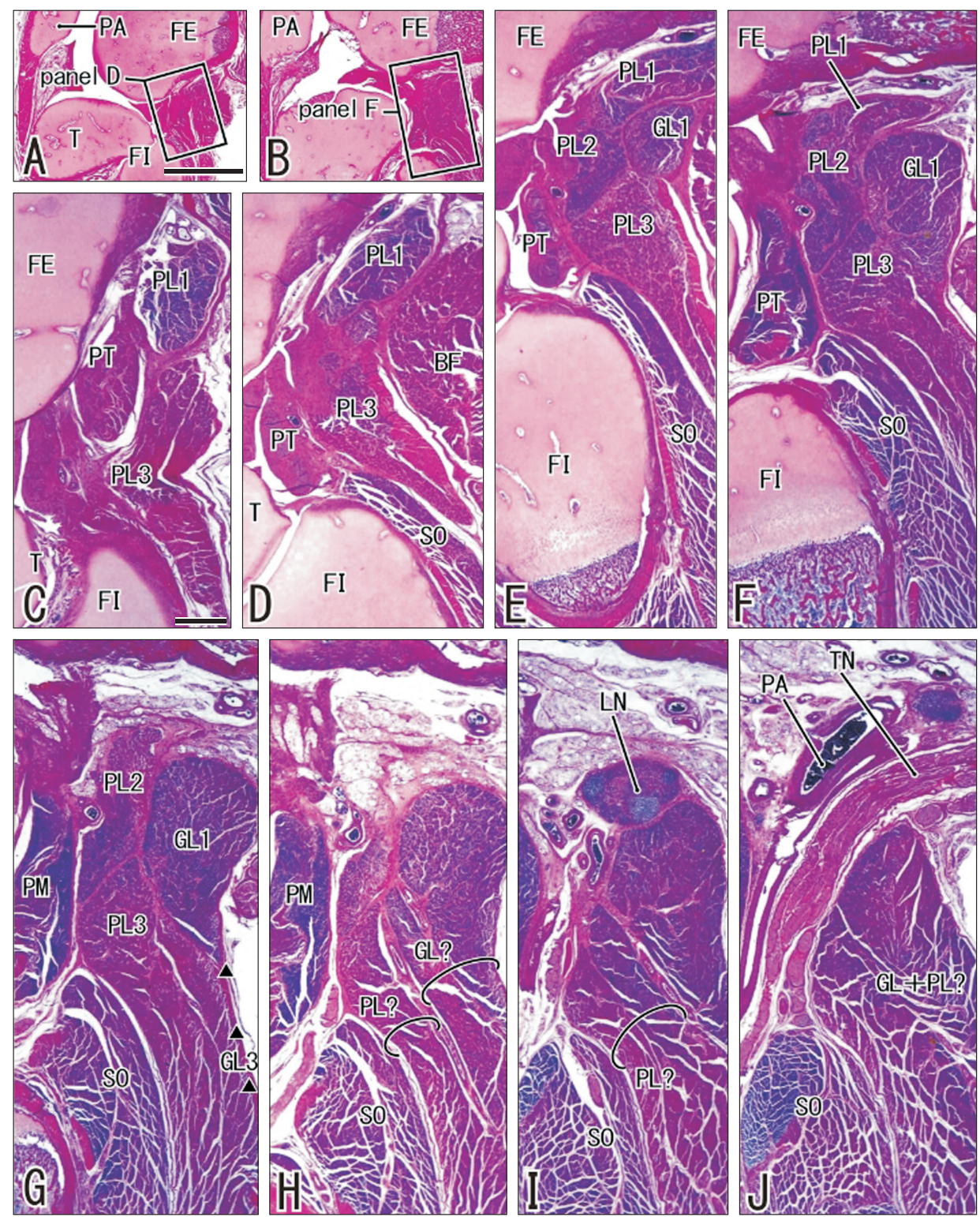

Fig. 5. Lack of a common tendinous origin of the gastrocnemius lateral head (GL) and plantaris (PL) in a fetus (274 mm crown-rump length [CRL]) without a fabella. (A, B) show the topographical anatomy of bony elements and muscle attachments in the lateral half of the knee joint. The squares in (A, B) are at a higher magnification in (D, F), respectively. (C-J) show multiple muscle bundles at the origins of these two muscles; (C) is shows the most lateral site and (J) the most medial site. (E, F) show the three muscle bundles at the PL origin (PL1, PL2, and PL3) provide a belt on the immediate posterior side of the popliteus tendon. (G) shows that the GL1 attaches to the posterior aspect of the PL origins and the GL3 originates from a surface aponeurosis from the GL1 (triangles). (A, B) are at low magnification (H\&E, scale bar in A: 5 mm) and (C$\mathrm{J}$ ) are at high magnification (H\&E, scale bar in C: $1 \mathrm{~mm}$ ). See also Table 1. PA, patella; BF, biceps femoris; FI, fibula; T, tibia; FE, femur; PM, popliteus muscle; PT, popliteus tendon; SO, soleus; LN, lymph node; TN, tibial nerve.

from the GL1 (Fig. 5G). We did not identify the GL2. Thus, this fetus had two major muscle bundles (PL1+PL2+PL3 and GL1+GL3) and a thick artery between these muscles (Fig. $5 \mathrm{H}$ ), although medial sections indicated both muscles were fused (Fig. 5J). The medial head of the gastrocnemius appeared normal on the medial side along the course of the tibial nerve.

\section{Discussion}

The most striking finding of our examination of late-term fetuses (28-37 weeks GA) was the presence of a complex of 
Table 1. Terms used in this study

\begin{tabular}{|c|c|}
\hline Terms & Features \\
\hline $\begin{array}{l}\text { "Common tendon" of the gastrocnemius lateral } \\
\text { head and plantaris in late-stage fetuses }\end{array}$ & $\begin{array}{l}\text { A thick tendon originating from the lateral condyle of the femur (similar to the adult morphology of the } \\
\text { lateral head) }\end{array}$ \\
\hline Fabella or its analogue & A spherical cartilage or a tight fibrous mass embedded in the aforementioned common tendon \\
\hline \multicolumn{2}{|l|}{ Plantaris (PL) } \\
\hline Upper bundle (PL1) & $\begin{array}{l}\text { From the lateral condyle in the immediately superoposterior side of the common tendon origin (similar to } \\
\text { the adult plantaris origin) }\end{array}$ \\
\hline Middle bundle (PL2) & From the poseteromedial aspect of the fabella or its analogue \\
\hline Lower bundle (PL3) & From the inferior aspect of the fabella or the inferomedial aspect of the fabella-analogue \\
\hline \multicolumn{2}{|l|}{ Gastrocnemius lateral head (GL) } \\
\hline Upper bundle (GL1) & From the common tendon in the superior side of the fabella or its analogue \\
\hline Middle bundle (GL2) & From the inferior aspect of the fabella or its analogue \\
\hline Lower bundle (GL3) & From an aponeurosis extending inferiorly from the posterior aspect of the GL2 \\
\hline
\end{tabular}

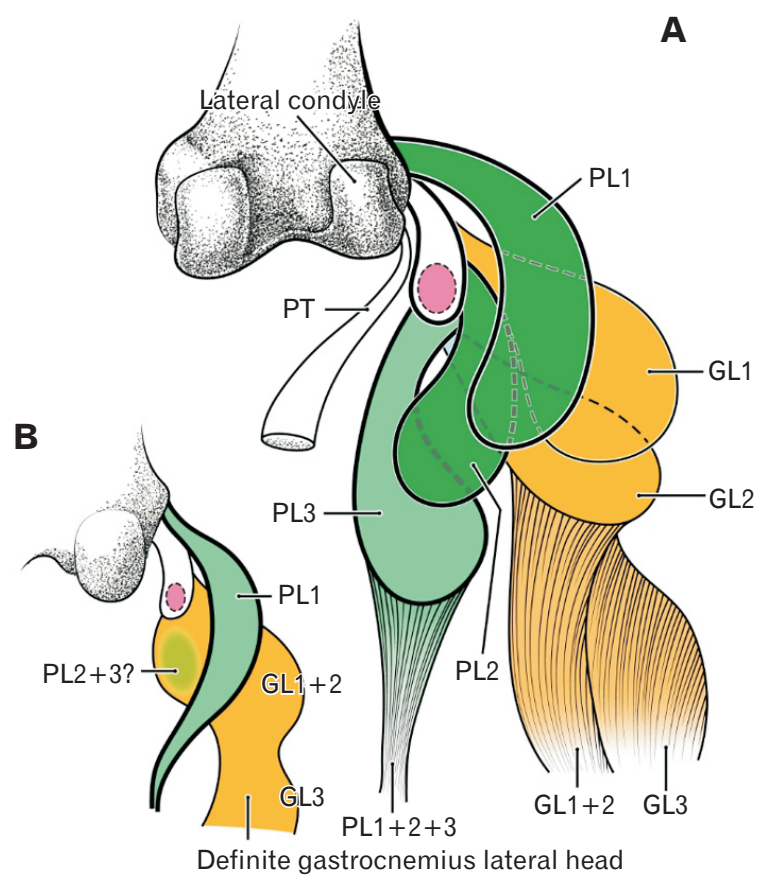

Fig. 6. Schematic representation of the gastrocnemius lateral head (GL) and plantaris (PL) origins in a late-term fetus, and hypothesized connections to adult morphology. The patella (or its analogue) is pink, the PL is green, and the GL is orange. (A) shows the topographical anatomy of the origins of the 3 GL bundles (GL1, GL2, and GL3) and the 3 PL bundles (PL1, PL2, and PL3). (B) shows the changes hypothesized to occur after birth, in which the PL1 remains, and the PL2 and PL3 degenerate or are absorbed into the gastrocnemius. PT, popliteus tendon.

muscle bundles at the origins of the GL and PL (Fig. 6A). This morphology is quite different from that in adults, in which a thick GL tendon often contains a bony fabella and the PL muscle fibers originate directly from the femur condyle. The PL muscle origin in adults is similar to that of the upper bundle of the PL (PL1) on the immediately superior side of the common tendon in the late-term fetuses examined here. Our previous study of midterm fetuses (15-18 weeks GA) indicated the PL origin appeared to contain a fabella or its analogue [1]. However, each midterm fetus had a dense connective tissue band containing a fabella or its analogue attached tightly to a PL muscle bundle; moreover, the GL and PL were attached to each other or even fused at the knee. Thus, at midterm, some muscle fibers of the GL may originate from the fabella or its analogue. The fibrous belt at midterm seemed to correspond to the common tendon at late-term, whereas the PL muscle bundle at midterm corresponded to PL1 at late-term. Thus the PL2 and PL3 appear later than the PL1. The present study of late-term fetuses indicated the fibrous belt was most likely to develop into a thick tendon (indicated here as the "common tendon") that provided origins of the GL1 and GL2 and of additional muscle bundles of the PL2 and PL3. Although we did not identify the G3 at midterm, the GL3 at late-term was similar to gastrocnemius muscle fibers originating from the surface aponeurosis below the knee.

After birth, drastic reconstructions presumably occur at the origins of the GL and PL. The common tendon was used only by the GL, whereas PL2 and PL3 degenerated or were absorbed into the GL (Fig. 6B). The prenatal space posterior to the knee joint is too narrow to contain all the growing ligaments, muscles, nerves, and blood vessels, because even a nerve makes a deep notch on the muscle surface $[1,6]$. Thus, the space occupying effect of the GL suggests that the multiple PL bundles at the origin are not maintained and do not grow during postnatal development.

Immediately after birth, the GL and vastus medialis play 
major roles in the "parachute reaction" of newborns [7]. In particular, the contact of an infant's foot with the floor (equinus foot without heel contact) is quite different from that in adults [8]. Moreover, when an infant starts walking, the gastrocnemius should act at all phases of the gait, because early walking consists simply of anterior foot attachment followed by toe-off, without complete plantar attachment and heelstrike, as in adults [9]. Early reflex and walking lead to much greater mechanical stress on the GL origin than the PL origin because the PL tendon is very thin in the Achilles tendon of late-term fetuses (not shown in the figures). We hypothesize that the great difference in muscle activity after birth may accelerate reconstruction or elimination of the multiple PL origins. These changes in the GL and PL are likely to be among the few examples of significant postnatal changes in skeletal muscle morphology.

One of the knees examined here differed from the other 14 knees, which each contained 3 transient origins of the GL and 3 transient origins of the PL. Because a muscle band of the PL1, PL2, and PL3 was evident in place of the common tendon and because a distal belly was absent from the PL, this fetal anomaly may give rise to a common variation in adults-an absent PL (variation No. 2, below). Bergman's Comprehensive Encyclopedia of Human Anatomic Variation [10] describes four variations in this region: (i) the PL muscle fibers often interdigitate with the GL at the origin (19.6\%); (ii) the PL is sometimes or often absent (2.5\%-13.0\%); (iii) a robust muscle belly equal to the GL is rarely reported in the PL; and (iv) the PL rarely originates from the GL. Absorption and/or degeneration of the entire fetal PL (PL1, PL2, and PL3) may thus explain the absence of a PL in adulthood. The morphology of the late-term fetus seems to be partly maintained by an interdigitation of muscle bundles of the GL and PL. For example, PL2, which was sandwiched by GL1 and GL2, was likey to be neither absorbed nor degenerated. The rare robust PL was identical to the late-term PL (Fig. 6A). Degeneration of the PL1 may have led to the anomalous PL arising from the GL (variation No. 4, above). That is, the PL was likely established during early evolution as a strong long flexor of the toes, but once the calcaneus was evident, the toe tendons were lost (reviewed by Jin et al. [1]). The presence of the PL in the toes was previously reported in kangaroos [11] and rabbits $[12,13]$. Finally, a well-known group of gastrocnemius variations (gastrocnemius tertius; $1.9-5.5 \%$ [10]) was likely to contain remnants of the PL2 or PL3.

A bony or cartilaginous fabella was reported to occur in $31.0 \%$ to $86.9 \%$ of tendinous origins of the GL in adults [14-17]. Our previous study of midterm fetuses [1] led us to hypothesize that the fabella initially develops at the origin of the PL. However, the present study of late-term fetuses showed that the fabella develops in the common tendon of the PL and GL. Later, possibly after birth, the fabella moves to a definite position depending on disappearance of the PL2 and PL3 origins from the common tendon. The development and growth of a tendon-associated fibrocartilage is an interesting aspect of fetal development $[18,19]$, and some studies investigated its genetic control, but most studies of sesamoid bones did not consider development of the fabella. Finally, our results lead us to reject the hypothesis that the patella originates from the femur and secondarily migrates into the GL [20].

\section{ORCID}

Zhe-Wu Jin: https://orcid.org/0000-0002-6789-2977

Ji Hyun Kim: https://orcid.org/0000-0002-9653-7261

Daisuke Suzuki: https://orcid.org/0000-0002-0162-7572

Namiko Sugai: https://orcid.org/0000-0002-3226-329X

Gen Murakami: https://orcid.org/0000-0001-7181-5475

Hiroshi Abe: https://orcid.org/0000-0003-2236-2929

José Francisco Rodríguez-Vázquez:

https://orcid.org/0000-0001-5423-4492

\section{Author Contributions}

Conceptualization: ZWJ, GM. Data acquisition: JHK, DS, NS, JFRV. Data analysis: ZWJ, JHK, GM, HA, JFRV. Drafting of the manuscript: ZWJ, JHK, GM. Critical revision of the manuscript: DS, NS, HA. Approval of the final version of the manuscript: all authors.

\section{Conflicts of Interest}

No potential conflict of interest relevant to this article was reported.

\section{References}

1. Jin ZW, Shibata S, Abe H, Jin Y, Li XW, Murakami G. A new insight into the fabella at knee: the foetal development and evolution. Folia Morphol (Warsz) 2017;76:87-93.

2. Rodríguez-Vázquez JF, Jin ZW, Zhao P, Murakami G, Li XW, 
Jin Y. Development of digastric muscles in human foetuses: a review and findings in the flexor digitorum superficialis muscle. Folia Morphol (Warsz) 2018;77:362-70.

3. Jin ZW, Jin Y, Yamamoto M, Abe H, Murakami G, Yan TF. Oblique cord (chorda obliqua) of the forearm and muscle-associated fibrous tissues at and around the elbow joint: a study of human foetal specimens. Folia Morphol (Warsz) 2016;75:493502.

4. Shiraishi Y, Jin ZW, Mitomo K, Yamamoto M, Murakami G, Abe H, Wilting J, Abe S. Foetal development of the human gluteus maximus muscle with special reference to its fascial insertion. Folia Morphol (Warsz) 2018;77:144-50.

5. Minowa T, Murakami G, Kura H, Suzuki D, Han SH, Yamashita $\mathrm{T}$. Does the fabella contribute to the reinforcement of the posterolateral corner of the knee by inducing the development of associated ligaments? J Orthop Sci 2004;9:59-65.

6. Nakamura T, Suzuki D, Murakami G, Cho BH, Fujimiya M, Kozuka N. Human fetal anatomy of the posterior semimembranosus complex at the knee with special reference to the gastrocnemio-semimembranosus bursa. Knee 2011;18:271-7.

7. Milani-Comparetti A, Gidoni EA. Routine developmental examination in normal and retarded children. Dev Med Child Neurol 1967;9:631-8.

8. Thelen E, Cooke DW. Relationship between newborn stepping and later walking: a new interpretation. Dev Med Child Neurol 1987;29:380-93.

9. Okamoto T, Okamoto K, Andrew PD. Electromyographic developmental changes in one individual from newborn stepping to mature walking. Gait Posture 2003;17:18-27.

10. Lambert HW. Leg muscles. In: Tubbs RS, Shoja MM, Loukas M, editors. Bergman's Comprehensive Encyclopedia of Human Anatomic Variation. Hoboken: John Wiley \& Sons; 2016. p.42137.
11. Warburton NM, Yakovleff M, Malric A. Anatomical adaptations of the hind limb musculature of tree-kangaroos for arboreal locomotion (Marsupialia: macropodinae). Aust J Zool 2012;60:246-58.

12. Doherty GP, Koike Y, Uhthoff HK, Lecompte M, Trudel G. Comparative anatomy of rabbit and human Achilles tendons with magnetic resonance and ultrasound imaging. Comp Med 2006;56:68-74.

13. Huisman ES, Andersson G, Scott A, Reno CR, Hart DA, Thornton GM. Regional molecular and cellular differences in the female rabbit Achilles tendon complex: potential implications for understanding responses to loading. J Anat 2014;224:538-47.

14. Kawashima T, Takeishi H, Yoshitomi S, Ito M, Sasaki H. Anatomical study of the fabella, fabellar complex and its clinical implications. Surg Radiol Anat 2007;29:611-6.

15. Phukubye $\mathrm{P}$, Oyedele $\mathrm{O}$. The incidence and structure of the fabella in a South African cadaver sample. Clin Anat 2011;24:8490.

16. Zeng SX, Dong XL, Dang RS, Wu GS, Wang JF, Wang D, Huang HL, Guo XD. Anatomic study of fabella and its surrounding structures in a Chinese population. Surg Radiol Anat 2012;34:65-71.

17. Chew CP, Lee KH, Koh JS, Howe TS. Incidence and radiological characteristics of fabellae in an Asian population. Singapore Med J 2014;55:198-201.

18. Benjamin M, Ralphs JR. Fibrocartilage in tendons and ligaments--an adaptation to compressive load. J Anat 1998;193(Pt 4):481-94.

19. Milz S, Benjamin M, Putz R. Molecular parameters indicating adaptation to mechanical stress in fibrous connective tissue. Adv Anat Embryol Cell Biol 2005;178:1-71.

20. Eyal S, Blitz E, Shwartz Y, Akiyama H, Schweitzer R, Zelzer E. On the development of the patella. Development 2015;142:1831-9. 